\title{
THE FOKKER-PLANCK-KOLMOGOROV EQUATIONS WITH A POTENTIAL AND A NON-UNIFORMLY ELLIPTIC DIFFUSION MATRIX
}

\author{
S. V. SHAPOSHNIKOV
}

\begin{abstract}
We study solutions of the Fokker-Planck-Kolmogorov equation with unbounded coefficients and a non-uniformly elliptic diffusion matrix. Upper bounds for solutions are obtained. In addition, new estimates with a Lyapunov function are obtained.
\end{abstract}

\section{$\S 1$. INTRODUCTION}

The purpose of this paper is to obtain upper bounds for solutions of the FokkerPlanck-Kolmogorov equation

$$
\partial_{t} \mu=\partial_{x_{i}} \partial_{x_{j}}\left(a^{i j} \mu\right)-\partial_{x_{i}}\left(b^{i} \mu\right)+c \mu .
$$

Below we use the summation convention, so there is summation over repeated indices. Given a number $T>0$, we say that a locally finite Borel measure $\mu$ on a set $\mathbb{R}^{d} \times(0, T)$ is defined by a flow of Borel measures $\left(\mu_{t}\right)_{t \in(0, T)}$. We use the notation $\mu=\mu_{t} d t$ if for every Borel set $B \subset \mathbb{R}^{d}$ the map $t \mapsto \mu_{t}(B)$ is measurable and the equation

$$
\int_{\mathbb{R}^{d} \times(0, T)} u(x, t) \mu(d x d t)=\int_{0}^{T} \int_{\mathbb{R}^{d}} u(x, t) \mu_{t}(d x) d t
$$

holds for every function $u \in C_{0}^{\infty}\left(\mathbb{R}^{d} \times(0, T)\right)$. A typical example is $\mu(B)=P\left(x_{t} \in B\right) d t$, where $x_{t}$ is a random process. We set

$$
L u=a^{i j} \partial_{x_{i}} \partial_{x_{j}} u+b^{i} \partial_{x_{i}} u+c u .
$$

We say that a measure $\mu=\left(\mu_{t}\right)_{t \in(0, T)}$ satisfies equation (1.1) if the functions $a^{i j}, b^{i}$, and $c$ are locally integrable with respect to the total variation $|\mu|$ of the measure $\mu$ and

$$
\int_{0}^{T} \int_{\mathbb{R}^{d}}\left[\partial_{t} u(x, t)+L u(x, t)\right] \mu_{t}(d x) d t=0
$$

for every function $u \in C_{0}^{\infty}\left(\mathbb{R}^{d} \times(0, T)\right)$. The measure $\mu$ satisfies the initial condition $\left.\mu\right|_{t=0}=\nu$, where $\nu$ is a locally finite Borel measure on $\mathbb{R}^{d}$, if

$$
\lim _{t \rightarrow 0} \int_{\mathbb{R}^{d}} \zeta(x) \mu_{t}(d x)=\int_{\mathbb{R}^{d}} \zeta(x) \nu(d x)
$$

for every function $\zeta \in C_{0}^{\infty}\left(\mathbb{R}^{d}\right)$.

2010 Mathematics Subject Classification. Primary 35R15; Secondary 35K10, 60J60.

Key words and phrases. Parabolic equations for measures, Fokker-Planck-Kolmogorov equation, diffusion processes.

This research was supported by the Russian Foundation for Basic Research (grant nos. 13-01-00332a, 11-01-00348-a, 12-01-33009, 11-01-12018-ofi-m-2011, 13-01-92100 JF) and Programme SFB 701 of Bielefeld University, Germany. 
We consider three classes of solutions: non-negative solutions, that is, locally finite Borel measures $\mu$ defined by a flow of non-negative measures $\mu_{t}$; sub-probability solutions, that is, finite Borel measures $\mu$ defined by a flow of non-negative measures $\mu_{t}$ such that $\mu_{t}\left(\mathbb{R}^{d}\right) \leq 1$ for almost all $t \in(0, T)$; and, finally, in the case $c \leq 0$, solutions $\mu$ defined by a flow of non-negative measures $\mu_{t}$ such that $|c| \in L^{1}(\mu)$ and

$$
\mu_{t}\left(\mathbb{R}^{d}\right) \leq \nu\left(\mathbb{R}^{d}\right)+\int_{0}^{t} \int_{\mathbb{R}^{d}} c(x, s) \mu_{s}(d x) d s
$$

for almost all $t \in(0, T)$.

We consider the class of solutions satisfying condition (1.2) separately. When the coefficients are globally bounded every solution $\mu$ defined by a family of sub-probability measures $\left(\mu_{t}\right)_{t \in(0, T)}$ satisfies (1.2). Furthermore, if the coefficient $c$ is a continuous function and $\mu_{t}$ is the weak limit of a sequence of measures $\mu_{t}^{n}$ satisfying (1.2), then $\mu_{t}$ also satisfies (1.2). Thus, this condition holds for every solution constructed as a weak limit of solutions of equations with bounded coefficients.

The main results in our paper consist of obtaining local and global $L^{p}$ and $L^{\infty}$ estimates for the densities of non-negative solutions of equation (1.1) with unbounded and non-uniformly elliptic coefficients, deriving a priori estimates with a Lyapunov function for solutions satisfying condition (1.2), and studying the behaviour of the densities of solutions in this class as $|x| \rightarrow+\infty$.

In the case when the matrix $A$ is uniformly elliptic, and the coefficients $a^{i j}, b^{i}$ and $c$ are bounded or have linear growth, estimates of Gaussian type are well known (see, for example, [5] and [8]). Global boundedness and upper bounds for the densities were obtained in [2] for solutions of Cauchy's problem for equation (1.1), in the case when the matrix $A$ is Lipschitz and uniformly elliptic but with no restrictions on the growth of the coefficients $b$ and $c$. This was done under the assumption that the initial condition is defined by the density and has finite entropy. In [9], 13, [14] and [16] the transition kernels of the semigroup $\left\{T_{t}\right\}$ were studied. For every continuous non-negative bounded function $f$ these define the minimal non-negative solution $T_{t} f$ of the Cauchy problem $\partial_{t} u=L u,\left.u\right|_{t=0}=f$. In the above papers it was assumed that the coefficients were independent of $t$ and locally Hölder continuous, that the diffusion matrix $A$ was uniformly elliptic and continuously differentiable, and that $c \leq 0$. In addition, the elements of the matrix $A$ and the coefficients $b, c$ had arbitrary growth as $|x| \rightarrow+\infty$. The main results in these papers establish upper estimates for the transition densities of the semigroup $\left\{T_{t}\right\}$ and the continuity of this semigroup in various function classes. Conditions were stated in terms of a Lyapunov function, for which a priori estimates independent of the initial condition were obtained. Note that the kernels of the semigroup $\left\{T_{t}\right\}$ satisfy equation (1.1), but the initial condition is Dirac's measure, and so the results in [2] cannot be applied.

In [6] and [7], estimates for the densities were obtained in the case of an arbitrary initial condition and the coefficient $b$ was only assumed to be locally integrable. However, the diffusion matrix was assumed to be uniformly bounded, uniformly elliptic and uniformly Lipschitz, and it was assumed that $c=0$. By contrast with the preceding papers, which used Moser's iteration technique to derive estimates over the whole of $\mathbb{R}^{d}$ directly, in 6 ] and [7] a new approach was proposed consisting of deriving global estimates from the local ones obtained in [11].

In all the above papers the assumption is made that $A(x, t) \geq \lambda I$ for all $(x, t) \in$ $\mathbb{R}^{d} \times(0, T)$ and some number $\lambda>0$. This assumption is essential in deriving global estimates. For example, in [2], 9], 13], 14, and [16] a priori estimates were derived using Moser's technique, and uniform ellipticity is of fundamental importance in this. We note that uniform ellipticity is not required for the existence of a solution, since the 
existence of the diffusion process corresponding to the operator $L$ only requires local regularity of the coefficients, and the transition probabilities of this diffusion process satisfy the Fokker-Planck-Kolmogorov equation (1.1). Therefore our main aim in this paper is to consider the case where the diffusion matrix can be both unbounded and non-uniformly elliptic. Using the ideas in [6] and 7] we derive global estimates from local ones, first making the dependence of the constants in the local estimates on the matrix $A$ more precise. Furthermore, the local estimates we obtain here are of interest in their own right and generalize the results in [11. Note that the conditions are stated in terms of the integrability of $A, b$ and $c$ with respect to the solution $\mu$ itself, rather than with respect to Lebesgue measure, which makes it possible to consider unbounded coefficients. A priori integrability of the coefficients with respect to a solution is verified using a Lyapunov function. Recall that a function $V \in C^{1,2}\left(\mathbb{R}^{d} \times(0, T)\right) \cap C\left(\mathbb{R}^{d} \times[0, T)\right)$ is called a Lyapunov function if the equation

$$
\lim _{|x| \rightarrow+\infty} \min _{t \in[a, b]} V(x, t)=+\infty
$$

holds for every closed interval $[a, b] \subset(0, T)$. Below we generalize the estimates using a Lyapunov function for the transition densities of a semigroup obtained in [14] and [16].

We look at an example which illustrates the estimates obtained here. Suppose that $d=d_{1}+d_{2} \geq 2$; we write $x=\left(x^{\prime}, x^{\prime \prime}\right)$, where $x^{\prime} \in \mathbb{R}^{d_{1}}, x^{\prime \prime} \in \mathbb{R}^{d_{2}}$. Let $r>2, k>r$, and $\delta \in(0,1)$. We set

$$
A(x, t)=e^{\left|x^{\prime}\right|^{r-\delta}-\left|x^{\prime \prime}\right|^{r-\delta}} I, \quad b(x, t)=-x|x|^{r-2} e^{\left|x^{\prime}\right|^{r-\delta}-\left|x^{\prime \prime}\right|^{r-\delta}}, \quad c(x, t)=-|x|^{k} .
$$

Then, if $\varrho$ is the density of a non-negative solution satisfying condition (1.2), the estimate

$$
\varrho(x, t) \leq c_{1} \exp \left(-c_{2}|x|^{r}\right) \exp \left(c_{3} t^{-r /(k-r)}\right)
$$

holds for all $(x, t) \in \mathbb{R}^{d} \times(0, T)$ and some positive numbers $c_{1}, c_{2}$ and $c_{3}$. Note that the estimate is independent of the initial condition $\nu$. In Example 3.9 at the end of the paper we consider a much more general case.

Lower bounds for solutions were obtained in [3]. The existence and uniqueness of a solution to the Cauchy problem for the Fokker-Planck-Kolmogorov equation were studied in [10] and [12. A detailed survey of research into elliptic and parabolic equations for measures was given in [1].

In the next section we consider a priori estimates with a Lyapunov function, and the last section of the paper is devoted to local and global estimates of solutions.

\section{$\S 2$. Estimates With a Lyapunov function}

Here we derive a priori estimates with a Lyapunov function, that is, we obtain sufficient conditions under which a Lyapunov function $V$ is integrable with respect to a solution $\mu$. We also obtain estimates for the integral

$$
\int_{\mathbb{R}^{d}} V(x, t) \mu_{t}(d x) .
$$

At the end of the section we give several examples with exponential and polynomial Lyapunov functions.

Recall that, given a diffusion process $x_{t}$ with generator $L$ and Lyapunov function $V$ for which the inequality $V_{t}+L V \leq C V$ holds for some number $C$, Itō's formula and Gronwall's inequality make it possible to obtain the following inequality:

$$
\mathbb{E} V\left(x_{t}, t\right) \leq \mathbb{E} V\left(x_{0}, 0\right) e^{C t} .
$$


We set $\mu_{t}(B):=P\left(x_{t} \in B\right)$. Then

$$
\mathbb{E} V\left(x_{t}, t\right)=\int_{\mathbb{R}^{d}} V(x, t) \mu_{t}(d x) .
$$

Below we derive similar estimates for solutions of the Fokker-Planck-Kolmogorov equation, but we do not use probabilistic methods, just the definition of a solution. In this case, restrictions on the coefficients are minimal. We assume that $c \leq 0$ and a solution $\mu$ is defined by a family of non-negative measures $\mu_{t}$ which satisfy (1.2), that is, $|c| \in L^{1}(\mu)$ and

$$
\mu_{t}\left(\mathbb{R}^{d}\right) \leq \nu\left(\mathbb{R}^{d}\right)+\int_{0}^{t} \int_{\mathbb{R}^{d}} c(x, s) \mu_{s}(d x) d s .
$$

In particular, the $\mu_{t}$ are sub-probability measures on $\mathbb{R}^{d}$. We do not assume any other conditions on the coefficients $a^{i j}, b^{i}$, and $c$. Note that the kernels considered in [13] satisfy (1.2).

The following auxiliary assertion was proved in [10], 12.

Lemma 2.1. Suppose that $\mu=\left(\mu_{t}\right)_{t \in(0, T)}$ is a solution of equation (1.1) and that a function $u$ in the class $C^{1,2}\left(\mathbb{R}^{d} \times(0, T)\right)$ is such that $u(x, t)=0$ for $x \notin U$ for some ball $U \subset \mathbb{R}^{d}$. Then there exists a set $J_{u} \subset(0, T)$ of full Lebesgue measure in $(0, T)$ such that

$$
\int_{\mathbb{R}^{d}} u(x, t) \mu_{t}(d x)=\int_{\mathbb{R}^{d}} u(x, s) \mu_{s}(d x)+\int_{s}^{t} \int_{\mathbb{R}^{d}}\left[\partial_{\tau} u(x, \tau)+L u(x, \tau)\right] \mu_{\tau}(d x) d \tau
$$

for all $s, t \in J_{u}$. Moreover, if $u \in C\left(\mathbb{R}^{d} \times[0, T)\right)$, the measure $\mu=\left(\mu_{t}\right)_{0<t<T}$ satisfies the initial condition $\left.\mu\right|_{t=0}=\nu$, and $a^{i j}, b^{i}, c \in L^{1}(U \times[0, T], \mu)$, then

$$
\int_{\mathbb{R}^{d}} u(x, t) \mu_{t}(d x)=\int_{\mathbb{R}^{d}} u(x, 0) \nu(d x)+\int_{0}^{t} \int_{\mathbb{R}^{d}}\left[\partial_{\tau} u(x, \tau)+L u(x, \tau)\right] \mu_{\tau}(d x) d \tau
$$

for all $t \in J_{u}$.

In the following theorem we derive an a priori estimate with a Lyapunov function.

Theorem 2.2. Suppose that $\mu=\left(\mu_{t}\right)_{0<t<T}$ is a solution of the Cauchy problem $\partial_{t} \mu=$ $L^{*} \mu,\left.\mu\right|_{t=0}=\nu$, where $c \leq 0$ and $\mu_{t}$, $\nu$ are sub-probability measures on $\mathbb{R}^{d}$ satisfying (1.2). Suppose that there exists a Lyapunov function $V$ such that, for some functions $K, H \in$ $L^{1}((0, T))$ with $H \geq 0$ and for all $(x, t) \in \mathbb{R}^{d} \times(0, T)$, we have the inequality

$$
\partial_{t} V(x, t)+L V(x, t) \leq K(t)+H(t) V(x, t) .
$$

Suppose also that $V(\cdot, 0) \in L^{1}(\nu)$. Then for almost all $t \in(0, T)$ we have

$$
\begin{gathered}
\mu_{t}\left(\mathbb{R}^{d}\right)=\nu\left(\mathbb{R}^{d}\right)+\int_{0}^{t} \int_{\mathbb{R}^{d}} c(x, s) \mu_{s}(d x) d s, \\
\int_{\mathbb{R}^{d}} V(x, t) \mu_{t}(d x) \leq Q(t)+R(t) \int_{\mathbb{R}^{d}} V(x, 0) \nu(d x),
\end{gathered}
$$

where

$$
R(t)=\exp \left(\int_{0}^{t} H(s) d s\right), \quad Q(t)=R(t) \int_{0}^{t} \frac{K(s)}{R(s)} d s
$$

Proof. Let the function $\zeta_{N} \in C^{2}([0,+\infty))$ be such that $0 \leq \zeta_{N}^{\prime} \leq 1, \zeta_{N}^{\prime \prime} \leq 0, \zeta_{N}(s)=s$ for $s \leq N-1$, and $\zeta_{N}(s)=N$ for $s>N+1$. Substituting the function $u=\zeta_{N}(V)-N$ 
into the equation in Lemma 2.1 we obtain

$$
\begin{aligned}
\int_{\mathbb{R}^{d}} \zeta_{N}(V(x, t)) \mu_{t}(d x)= & \int_{\mathbb{R}^{d}} \zeta_{N}(V(x, s)) \mu_{s}(d x) \\
& +\left(\mu_{t}\left(\mathbb{R}^{d}\right)-\nu\left(\mathbb{R}^{d}\right)-\int_{s}^{t} \int_{\mathbb{R}^{d}} c(x, \tau) \mu_{\tau}(d x) d \tau\right) N \\
& +\int_{s}^{t} \int_{\mathbb{R}^{d}}\left(\zeta_{N}^{\prime}(V)\left(\partial_{t} V+L V\right)+\zeta_{N}^{\prime \prime}(V)|\sqrt{A} \nabla V|^{2}\right) \mu_{\tau}(d x) d \tau \\
& +\int_{s}^{t} \int_{\mathbb{R}^{d}} c\left(\zeta_{N}(V)-\zeta_{N}^{\prime}(V) V\right) \mu_{\tau}(d x) d \tau .
\end{aligned}
$$

Note that $z \zeta_{N}^{\prime}(z) \leq \zeta_{N}(z)$. Therefore,

$$
\begin{aligned}
& \int_{\mathbb{R}^{d}} \zeta_{N}(V(x, t)) \mu_{t}(d x) \leq \int_{\mathbb{R}^{d}} \zeta_{N}(V(x, s)) \mu_{s}(d x) \\
&+\left(\mu_{t}\left(\mathbb{R}^{d}\right)-\nu\left(\mathbb{R}^{d}\right)-\int_{s}^{t} \int_{\mathbb{R}^{d}} c(x, \tau) \mu_{\tau}(d x) d \tau\right) N \\
& \quad+\int_{s}^{t}\left(K(\tau)+H(\tau) \int_{\mathbb{R}^{d}} \zeta_{N}(V(x, \tau)) \mu_{\tau}(d x)\right) d \tau .
\end{aligned}
$$

Letting $s \rightarrow 0$ we arrive at the inequality

$$
\begin{aligned}
& \int_{\mathbb{R}^{d}} \zeta_{N}(V(x, t)) d \mu_{t} \leq \int_{\mathbb{R}^{d}} \zeta_{N}(V(x, 0)) d \nu \\
&+\left(\mu_{t}\left(\mathbb{R}^{d}\right)-\nu\left(\mathbb{R}^{d}\right)-\int_{0}^{t} \int_{\mathbb{R}^{d}} c(x, \tau) \mu_{\tau}(d x) d \tau\right) N \\
& \quad+\int_{0}^{t}\left(K(\tau)+H(\tau) \int_{\mathbb{R}^{d}} \zeta_{N}(V(x, \tau)) \mu_{\tau}(d x)\right) d \tau
\end{aligned}
$$

Since

$$
\mu_{t}\left(\mathbb{R}^{d}\right) \leq \nu\left(\mathbb{R}^{d}\right)+\int_{0}^{t} \int_{\mathbb{R}^{d}} c(x, \tau) \mu_{\tau}(d x) d \tau,
$$

the last inequality can be rewritten in the following form:

$$
\begin{aligned}
\int_{\mathbb{R}^{d}} \zeta_{N}(V(x, t)) \mu_{t}(d x) \leq \int_{\mathbb{R}^{d}} \zeta_{N}(V(x, 0)) \nu(d x) & \\
& +\int_{0}^{t}\left(K(\tau)+H(\tau) \int_{\mathbb{R}^{d}} \zeta_{N}(V(x, \tau)) \mu_{\tau}(d x)\right) d \tau .
\end{aligned}
$$

Applying Gronwall's inequality we obtain

$$
\int_{\mathbb{R}^{d}} \zeta_{N}(V(x, t)) \mu_{t}(d x) \leq Q(t)+R(t) \int_{\mathbb{R}^{d}} \zeta_{N}(V(x, 0)) \nu(d x) .
$$

Letting $N \rightarrow \infty$ we obtain the required estimate. Note that if

$$
\mu_{t}\left(\mathbb{R}^{d}\right)<\nu\left(\mathbb{R}^{d}\right)+\int_{0}^{t} \int_{\mathbb{R}^{d}} c(x, \tau) \mu_{\tau}(d x) d \tau,
$$

then

$$
\begin{aligned}
\int_{\mathbb{R}^{d}} V(x, t) \mu_{t}(d x)-\int_{\mathbb{R}^{d}} V(x, 0) & \nu(d x) \\
& -\int_{0}^{t}\left(K(\tau)+H(\tau) \int_{\mathbb{R}^{d}} V(x, \tau) \mu_{\tau}(d x)\right) d \tau=-\infty,
\end{aligned}
$$


which is impossible. Thus, we have the equation

$$
\mu_{t}\left(\mathbb{R}^{d}\right)=\nu\left(\mathbb{R}^{d}\right)+\int_{0}^{t} \int_{\mathbb{R}^{d}} c(x, \tau) \mu_{\tau}(d x) d \tau .
$$

The theorem is proved.

Corollary 2.3. Suppose that $\mu=\left(\mu_{t}\right)_{0<t<T}$ is a solution of the Cauchy problem $\partial_{t} \mu=$ $L^{*} \mu,\left.\mu\right|_{t=0}=\nu$, where $c \leq 0$ and $\mu_{t}, \nu$ are sub-probability measures on $\mathbb{R}^{d}$ satisfying condition (1.2). Suppose that the positive function $W \in C^{2}\left(\mathbb{R}^{d}\right)$ is such that

$$
\lim _{|x| \rightarrow+\infty} W(x)=+\infty .
$$

(i) If for some number $C>0$ and almost all $(x, t) \in \mathbb{R}^{d} \times(0, T)$ the inequality

$$
L W(x, t) \leq C+C W(x)
$$

holds, then the estimate

$$
\int_{\mathbb{R}^{d}} W(x) \mu_{t}(d x) \leq \exp (C t)+\exp (C t) \int_{\mathbb{R}^{d}} W(x) \nu(d x)
$$

holds for almost all $t \in(0, T)$.

(ii) Suppose that $G$ is a positive continuous increasing function on $[0,+\infty)$ such that

$$
\int_{1}^{+\infty} \frac{d s}{s G(s)}<+\infty
$$

Let $\eta$ be the continuous function on $[0, T)$ defined by the equation

$$
t=\int_{0}^{\eta(t)} \frac{d s}{s G\left(s^{-\delta}\right)}, \quad \delta \in(0,1) .
$$

If the inequality

$$
L W(x, t) \leq C-W(x) G(W(x))
$$

holds for some number $C>0$ and all $(x, t) \in \mathbb{R}^{d} \times(0, T)$, then

$$
\int_{\mathbb{R}^{d}} W(x) \mu_{t}(d x) \leq \frac{1}{(1-\delta) \eta^{\delta}(t)}+\frac{C}{\eta(t)} \int_{0}^{t} \eta(s) d s
$$

for almost all $t \in(0, T)$.

(iii) Let $G$ and $\eta$ be the functions defined in (ii). Suppose that the inequality

$$
L W(x, t)+\eta(t)|\sqrt{A(x, t)} \nabla W(x)|^{2} \leq C-W(x) G(W(x))
$$

holds for some number $C>0$ and all $(x, t) \in \mathbb{R}^{d} \times(0, T)$. Then

$$
\int_{\mathbb{R}^{d}} \exp (\eta(t) W(x)) \mu_{t}(d x) \leq \exp \left((1-\delta)^{-1} \eta^{1-\delta}(t)+C \int_{0}^{t} \eta(s) d s\right)
$$

for almost all $t \in(0, T)$.

Proof. To prove (i) it suffices to apply Theorem 2.2 with the functions $H(t)=K(t)=C$ and $V(x, t)=W(x)$.

We now prove (ii). We set $V(x, t)=\eta(t) W(x)$. Then

$$
\partial_{t} V(x, t)+L V(x, t) \leq \eta^{\prime}(t) W(x)-\eta(t) W(x) G(W(x))+C \eta(t) .
$$

We observe that the inequality

$$
\alpha \beta \leq \alpha G^{-1}(\alpha)+\beta G(\beta)
$$


holds for all non-negative numbers $\alpha$ and $\beta$, where $G^{-1}$ is the inverse function of $G$. Using this inequality with $\alpha=\eta^{\prime} / \eta$ and $\beta=W$ we obtain

$$
\partial_{t} V(x, t)+L V(x, t) \leq \eta^{\prime}(t) G^{-1}\left(\frac{\eta^{\prime}(t)}{\eta(t)}\right)+C \eta(t)=\frac{\eta^{\prime}(t)}{\eta^{\delta}(t)}+C \eta(t),
$$

since $\eta^{\prime}(t)=\eta(t) G\left(\eta^{-\delta}(t)\right)$ by hypothesis.

Applying Theorem 2.2 with $H(t)=0$ and $K(t)=\eta^{\prime}(t) / \eta^{\delta}(t)+C \eta(t)$, we obtain the required inequality.

We now prove (iii). Let $V(x, t)=\exp (\eta(t) W(x))$. Then

$$
\partial_{t} V(x, t)+L V(x, t) \leq\left[\eta^{\prime}(t) W(x)-\eta(t) W(x) G(W(x))+C \eta(t)\right] \exp (\eta(t) W(x)) .
$$

Consequently,

$$
\partial_{t} V(x, t)+L V(x, t) \leq\left[\frac{\eta^{\prime}(t)}{\eta^{\delta}(t)}+C \eta(t)\right] \exp (\eta(t) W(x)) .
$$

Applying Theorem 2.2 with $K(t)=0$ and

$$
H(t)=\frac{\eta^{\prime}(t)}{\eta^{\delta}(t)}+C \eta(t)
$$

we arrive at the required assertion.

Now we look at some examples.

Example 2.4. Let $V(x, t)=|x|^{r}$, where $r \geq 2$. Then

$$
\begin{aligned}
L V(x, t)=r|x|^{r-2} \operatorname{tr} A(x, t)+r(r-2)|x|^{r-4}(A(x, t) x, x) & \\
& +r|x|^{r-2}(b(x, t), x)+|x|^{r} c(x, t) .
\end{aligned}
$$

Suppose that the inequality

$$
r \operatorname{tr} A(x, t)+r(r-2)|x|^{-2}(A(x, t) x, x)+r(b(x, t), x)+|x|^{2} c(x, t) \leq C_{1}+C_{2}|x|^{2}
$$

holds for some numbers $C_{1}>0, C_{2}>0$ and all $(x, t) \in \mathbb{R}^{d} \times[0, T]$. Let $|x|^{r} \in L^{1}(\nu)$. Then

$$
\int_{\mathbb{R}^{d}}|x|^{r} \mu_{t}(d x) \leq e^{C_{3} t}+e^{C_{3} t} \int_{\mathbb{R}^{d}}|x|^{r} \nu(d x)
$$

for almost all $t \in(0, T)$ and some number $C_{3}>0$.

Example 2.5. Let $V(x, t)=\exp \left(\alpha|x|^{r}\right)$, where $r \geq 2$. Then

$$
\begin{aligned}
& \quad L V(x, t)=\exp \left(\alpha|x|^{r}\right)\left[\alpha r|x|^{r-2} \operatorname{tr} A(x, t)+\alpha r(r-2)|x|^{r-4}(A(x, t) x, x)\right. \\
& \left.\quad+\alpha^{2} r^{2}|x|^{2 r-4}(A(x, t) x, x)+\alpha r|x|^{r-2}(b(x, t), x)+c(x, t)\right] .
\end{aligned}
$$

Suppose that the inequality

$$
\begin{aligned}
\alpha r|x|^{r-2} \operatorname{tr} A(x, t)+ & \alpha r(r-2)|x|^{r-4}(A(x, t) x, x) \\
& +\alpha^{2} r^{2}|x|^{2 r-4}(A(x, t) x, x)+\alpha r|x|^{r-2}(b(x, t), x)+c(x, t) \leq C_{1}
\end{aligned}
$$

holds for some number $C_{1}$ and for all $(x, t) \in \mathbb{R}^{d} \times[0, T]$.

If $\exp \left(|x|^{r}\right) \in L^{1}(\nu)$, then

$$
\int_{\mathbb{R}^{d}} \exp \left(\alpha|x|^{r}\right) \mu_{t}(d x) \leq e^{C_{2} t}+e^{C_{2} t} \int_{\mathbb{R}^{d}} \exp \left(\alpha|x|^{r}\right) \nu(d x)
$$

for almost all $t \in(0, T)$ and some positive number $C_{2}$. 
Example 2.6. Let $k>2$ and $r \geq 2$. Suppose that for all $(x, t) \in \mathbb{R}^{d} \times(0, T)$ and some numbers $C_{1}>0$ and $C_{2}>0$,

$$
r \operatorname{tr} A(x, t)+r(r-2)|x|^{-2}(A(x, t) x, x)+r(b(x, t), x)+|x|^{2} c(x, t) \leq C_{1}-C_{2}|x|^{k} .
$$

Then

$$
L|x|^{r} \leq C_{3}-C_{3}|x|^{r+k-2}
$$

for some number $C_{3}>0$. Set $W(x)=|x|^{r}$ and $G(z)=C_{3} z^{\sigma}$, where $\sigma=\frac{k-2}{r}>0$. Then

$$
L W(x, t) \leq C_{3}-W G(W(x))
$$

and $\eta(t)=C_{4} t^{1 /(\delta \sigma)}$, where $C_{4}$ depends only on $C_{3}, \delta$, and $\sigma$. Applying Corollary 2.3 we obtain the estimate

$$
\int_{\mathbb{R}^{d}}|x|^{r} \mu_{t}(d x) \leq \frac{\gamma}{t^{r /(k-2)}}
$$

where $\gamma$ depends on $C_{1}, C_{2}, \delta, \sigma$.

Example 2.7. Let $r>2$ and $k>r$. Suppose that the inequality

$$
\begin{aligned}
\alpha r|x|^{r-2} \operatorname{tr} A(x, t)+\alpha r(r-2)|x|^{r-4}(A(x, t) x, x)+\alpha^{2} r^{2}|x|^{2 r-4}(A(x, t) x, x) \\
+\alpha r|x|^{r-2}(b(x, t), x)+c(x, t) \leq C_{1}-C_{2}|x|^{k}
\end{aligned}
$$

holds for all $(x, t) \in \mathbb{R}^{d} \times(0, T)$ and some numbers $C_{1}>0$ and $C_{2}>0$. Then

$$
L \exp \left(\alpha|x|^{r}\right) \leq C_{3}-C_{3}|x|^{k} \exp \left(\alpha|x|^{r}\right)
$$

for some $C_{3}>0$. We set $W(x)=\exp \left(\alpha|x|^{r}\right)$ and $G(z)=C_{3}|\ln z|^{\sigma}$ if $z \geq 2$, where $\sigma=k / r>1$. We obtain

$$
L W(x, t) \leq C_{3}-W G(W(x))
$$

and $\eta(t)=C_{4} \exp \left(-C_{5} t^{-1 /(\sigma-1)}\right)$, where $C_{4}>0$ and $C_{5}>0$ depend only on $C_{3}, \delta$, and $\sigma$. Applying Corollary 2.3 we arrive at the inequality

$$
\int_{\mathbb{R}^{d}} \exp \left(\alpha|x|^{r}\right) \mu_{t}(d x) \leq \gamma_{1} \exp \left(\frac{\gamma_{2}}{t^{r /(k-r)}}\right),
$$

where $\gamma_{1}$ and $\gamma_{2}$ depend only on $C_{1}, C_{2}, \delta$, and $\sigma$.

Example 2.8. Let $r>2, k>2$, and $\alpha>0$. Suppose that the inequality

$$
\begin{aligned}
\alpha r \operatorname{tr} A(x, t)+ & \alpha r(r-2)|x|^{-2}(A(x, t) x, x) \\
& +\alpha r(b(x, t), x)+\alpha|x|^{2} c(x, t)+\alpha^{2} r^{2}|x|^{r-2}(A(x, t) x, x) \leq C_{1}-C_{2}|x|^{k}
\end{aligned}
$$

holds for all $(x, t) \in \mathbb{R}^{d} \times(0, T)$ and some numbers $C_{1}>0$ and $C_{2}>0$. Then

$$
\alpha L|x|^{r}+\alpha^{2} r^{2}|x|^{2 r-4}(A(x, t) x, x) \leq C_{3}-C_{3}|x|^{k+r-2} .
$$

Let $W(x)=\alpha|x|^{r}$ and $G(z)=C_{3} \alpha^{-(1+\sigma) / \sigma} z^{\sigma}$, where $\sigma=\frac{k-2}{r}>0$. Then

$$
L W(x, t)+|\sqrt{A(x, t)} \nabla W(x)|^{2} \leq C_{3}-W G(W(x)) .
$$

Applying Corollary 2.3 with $\delta \in(0,1)$ and $\eta(t)=C_{4} t^{1 /(\delta \sigma)}$, where $C_{4}$ depends only on $C_{3}, \delta$, and $\sigma$, for all $\beta>r /(k-2)$ we obtain the estimate

$$
\int_{\mathbb{R}^{d}} \exp \left(\alpha t^{\beta}|x|^{r}\right) \mu_{t}(d x) \leq \gamma_{1} \exp \left(\gamma_{2}\left(t^{\beta-r /(k-2)}+t^{\beta+1}\right)\right),
$$

where the positive numbers $\gamma_{1}$ and $\gamma_{2}$ depend only on $C_{1}, C_{2}, r$, and $\beta$. 
Note that the estimates in Examples 2.7 and 2.8 are independent of the initial condition. Applying the results of these examples to the transition probabilities $P(y, 0, t, d x)$ of a diffusion process with generator $L$ we can obtain estimates that are uniform in $y$. As we already mentioned above, the first estimates of this type were obtained in [14 and [16] for semigroup kernels.

\section{$\S$ 3. Local AND GLOBAL ESTimates of SOlutions}

In this section we derive local and global $L^{p}$ and $L^{\infty}$ estimates for the densities of solutions. First we derive local estimates using Moser's iteration technique (see [15]), and then obtain global estimates using a suitable scaling.

Let $\mu=\left(\mu_{t}\right)_{t \in(0, T)}$ be a non-negative solution of equation (1.1).

We assume that $A=\left(a^{i j}\right)$ is a symmetric matrix satisfying the following conditions:

(H1) for some $p>d+2$, for every ball $U \subset \mathbb{R}^{d}$ and every closed interval $J \subset(0, T)$, we have

$$
\begin{gathered}
\sup _{t \in J}\left\|a^{i j}(\cdot, t)\right\|_{W^{1, p}(U)}<\infty, \\
0<\lambda(U, J):=\inf \{(A(x, t) \xi, \xi):|\xi|=1,(x, t) \in U \times J\} .
\end{gathered}
$$

Furthermore, we assume that

(H2) for the number $p>d+2$ in condition (H1), for every ball $U \subset \mathbb{R}^{d}$ and every closed interval $J \subset(0, T)$, we have

$$
b, c \in L^{p}(U \times J) \quad \text { or } \quad b, c \in L^{p}(U \times J, \mu) .
$$

By [11, Corollary 3.9] and [4, Corollary 2.2], conditions (H1) and (H2) guarantee the existence of a Hölder continuous density $\varrho$ of a solution $\mu$ with respect to Lebesgue measure. Moreover, for every ball $U \subset \mathbb{R}^{d}$ and every closed interval $J \subset(0, T)$ we have the inclusion $\varrho(\cdot, t) \in W^{1, p}(U)$ and

$$
\int_{J}\|\varrho(\cdot, t)\|_{W^{1, p}(U)}^{p} d t<\infty
$$

We set $B^{i}=b^{i}-\partial_{x_{j}} a^{i j}$. Then equation (1.1) can be rewritten in the form of a parabolic equation in divergence form

$$
\partial_{t} \varrho=\operatorname{div}(A \nabla \varrho-B \varrho)+c \varrho,
$$

which is understood in the sense of the integral identity

$$
\int_{0}^{T} \int_{\mathbb{R}^{d}}\left[-\varrho \partial_{t} \varphi+(A \nabla \varrho, \nabla \varphi)\right] d x d t=\int_{0}^{T} \int_{\mathbb{R}^{d}}[(B, \nabla \varphi) \varrho+c \varrho \varphi] d x d t
$$

for every function $\varphi \in C_{0}^{\infty}\left(\mathbb{R}^{d} \times(0, T)\right)$.

Recall the following embedding theorem (see [2, Lemma 3.1] or [8]).

Lemma 3.1. Let $J$ be a closed interval in $(0, T)$ and suppose that a function $u(\cdot, t) \in$ $W^{1,2}\left(\mathbb{R}^{d}\right)$ is such that the map $x \mapsto u(x, t)$ has compact support for almost all $t \in J$. Then there exists a number $C>0$ depending only on the dimension $d$ such that

$$
\|u\|_{L^{2(d+2) / d}\left(\mathbb{R}^{d} \times J\right)} \leq C\left(\sup _{t \in J}\|u(\cdot, t)\|_{L^{2}\left(\mathbb{R}^{d}\right)}+\|\nabla u\|_{L^{2}\left(\mathbb{R}^{d} \times J\right)}\right) .
$$

Note that we now do not assume that $c \leq 0$.

We set $c^{+}(x, t)=\max \{c(x, t), 0\}$.

The following lemma is a key result in this paper. 
Lemma 3.2. Let $m \geq 1$, let $U \subset \mathbb{R}^{d}$ be an arbitrary ball, and let $\left[s_{1}, s_{2}\right] \subset(0, T)$. Suppose that a function $\psi \in C_{0}^{\infty}\left(\mathbb{R}^{d} \times(0, T)\right)$ is such that its support is contained in $U \times(0, T)$ and $\psi\left(x, s_{1}\right)=0$ for all $x$. Then there exists a number $C(d)>0$ depending only on $d$ such that

$$
\begin{aligned}
\left(\int_{s_{1}}^{s_{2}} \int_{U}\left|\varrho^{m} \psi\right|^{2(d+2) / d} d x d t\right)^{d /(d+2)} & \leq 32 C(d) m^{2}\left(1+\lambda^{-1}\right) \\
& \times \int_{s_{1}}^{s_{2}} \int_{U}\left(|\psi| \cdot\left|\psi_{t}\right|+\|A\| \cdot|\nabla \psi|^{2}+\left|\sqrt{A^{-1}} B\right|^{2} \psi^{2}+c^{+} \psi^{2}\right) \varrho^{2 m} d x d t
\end{aligned}
$$

where $\|A(x, t)\|=\max _{|\xi|=1}(A(x, t) \xi, \xi)$ and the number $\lambda=\lambda\left(U,\left[s_{1}, s_{2}\right]\right)$ is defined above.

Proof. Let $f$ be a smooth function on $[0,+\infty)$ such that $f \geq 0, f^{\prime} \geq 0, f^{\prime \prime} \geq 0$. Substituting the function $\varphi=f^{\prime}(\varrho) \psi^{2}$ into the identity (3.2), for every $t \in\left[s_{1}, s_{2}\right]$ we obtain the inequality

$$
\begin{aligned}
\int_{\mathbb{R}^{d}} f(\varrho(x, t)) \psi^{2}(x) d x-\int_{\mathbb{R}^{d}} f\left(\varrho\left(x, s_{1}\right)\right) \psi^{2}(x) d x & \\
& +\frac{1}{3} \int_{s_{1}}^{t} \int_{\mathbb{R}^{d}}|\sqrt{A} \nabla \varrho|^{2} f^{\prime \prime}(\varrho) \psi^{2} d x d \tau \\
\leq \int_{s_{1}}^{t} \int_{\mathbb{R}^{d}} 2|\psi| \cdot\left|\psi_{t}\right| f(\varrho)+3|\sqrt{A} \nabla \psi|^{2} & \frac{f^{\prime}(\varrho)^{2}}{f^{\prime \prime}(\varrho)}+3\left|\sqrt{A^{-1}} B\right|^{2} \varrho^{2} f^{\prime \prime}(\varrho) \psi^{2} \\
& +2|(B, \nabla \psi)| \psi \varrho f^{\prime}(\varrho)+c^{+} \varrho f^{\prime}(\varrho) \psi^{2} d x d \tau .
\end{aligned}
$$

In fact, it is enough to observe that

$$
\begin{aligned}
& 2(A \nabla \varrho, \nabla \psi) \psi f^{\prime}(\varrho) \leq 3^{-1}|\sqrt{A} \nabla \varrho|^{2} f^{\prime \prime}(\varrho) \psi^{2}+3|\sqrt{A} \nabla \psi|^{2} \frac{f^{\prime}(\varrho)^{2}}{f^{\prime \prime}(\varrho)} \\
& (B, \nabla \varrho) \varrho f^{\prime \prime}(\varrho) \psi^{2} \leq 3^{-1}|\sqrt{A} \nabla \varrho|^{2} f^{\prime \prime}(\varrho) \psi^{2}+3\left|\sqrt{A^{-1}} B\right|^{2} \varrho^{2} f^{\prime \prime}(\varrho) \psi^{2}
\end{aligned}
$$

We set $f(\varrho)=\varrho^{2 m}$. Recall that $\psi\left(x, s_{1}\right)=0$. We have

$$
\begin{aligned}
\sup _{t \in\left[s_{1}, s_{2}\right]} \int_{\mathbb{R}^{d}} \varrho^{2 m}(x, t) \psi^{2}(x) d x+\frac{4 m-2}{3 m} \int_{s_{1}}^{s_{2}} \int_{\mathbb{R}^{d}}\left|\sqrt{A} \nabla\left(\varrho^{m} \psi\right)\right|^{2} d x d \tau \\
\leq 32 m^{2} \int_{s_{1}}^{s_{2}} \int_{\mathbb{R}^{d}}\left(|\psi| \cdot\left|\psi_{t}\right|+|\sqrt{A} \nabla \psi|^{2}+\left|\sqrt{A^{-1}} B\right|^{2} \psi^{2}+c^{+} \psi^{2}\right) \varrho d x d \tau .
\end{aligned}
$$

Our assertion now follows from Lemma 3.1.

Theorem 3.3. Let $p \geq 2(d+2) / d$ and suppose that $U$ and $U^{\prime}$ are given balls in $\mathbb{R}^{d}$ such that $\overline{U^{\prime}} \subset U$. Also let $\left[s_{1}, s_{2}\right] \subset(0, T)$. Then for all $s \in\left(s_{1}, s_{2}\right)$ there exists a number $C>0$ depending only on $U, U^{\prime}, s, s_{1}, d$ and $p$ such that

$$
\|\varrho\|_{L^{p}\left(U^{\prime} \times\left[s, s_{2}\right]\right)} \leq C\left(1+\lambda^{-1}\right)^{\gamma} \int_{s_{1}}^{s_{2}} \int_{U}\left(1+\|A\|^{\gamma}+\left|c^{+}\right|^{\gamma}+\left|\sqrt{A^{-1}} B\right|^{2 \gamma}\right) \varrho d x d t,
$$

where $\gamma=(d+2) /\left(2 p^{\prime}\right), p^{\prime}=p /(p-1)$, and the numbers $\lambda=\lambda\left(U,\left[s_{1}, s_{2}\right]\right)$ and $\|A\|$ are defined above.

Proof. We set $m=d p /(2(d+2))$ and

$$
\alpha=1+\frac{4 m}{(2 m-1) d}, \quad \alpha^{\prime}=1+\frac{(2 m-1) d}{4 m}, \quad \delta=\frac{4}{d(2 m-1)+4 m} .
$$


Note that $m \geq 1$. We set $\psi=\zeta(x) \eta(t)$, where $\zeta \in C_{0}^{\infty}(U), \zeta(x)=1$ for $x \in U^{\prime}$, $0 \leq \psi \leq 1, \eta \in C_{0}^{\infty}\left(\left(s_{1}, T\right)\right), \eta(t)=1$ for $t \in\left[s, s_{2}\right], 0 \leq \eta \leq 1$, and

$$
\left|\partial_{t} \eta(t)\right| \leq K \eta^{1-\delta}(t), \quad|\nabla \zeta(x)| \leq K \zeta^{1-\delta}(x)
$$

for some $K>0$ and all $(x, t) \in U \times\left[s_{1}, s_{2}\right]$. Note that $K$ depends only on $U, U^{\prime}, s$, and $s_{1}$. Applying Lemma 3.2 we obtain

$$
\begin{aligned}
& \left(\int_{s_{1}}^{s_{2}} \int_{U}\left|\varrho^{m} \psi\right|^{2(d+2) / d} d x d t\right)^{d /(d+2)} \leq 32 C(d) m^{2}\left(1+\lambda^{-1}\right) \\
& \quad \times \int_{s}^{s_{2}} \int_{U}\left(|\psi| \cdot\left|\psi_{t}\right|+\|A\| \cdot|\nabla \psi|^{2}+\left|\sqrt{A^{-1}} B\right|^{2} \psi^{2}+c^{+} \psi^{2}\right) \varrho^{2 m} d x d t .
\end{aligned}
$$

Using Hölder's inequality with exponents $\alpha$ and $\alpha^{\prime}$ we can bound the integral on the right-hand side of the last inequality by the following expression:

$$
\begin{aligned}
K^{2}\left(\int_{s_{1}}^{s_{2}} \int_{U}\left(1+\|A\|+\left|\sqrt{A^{-1}} B\right|^{2}+c^{+}\right)^{\alpha^{\prime}} \varrho^{2 m} d x d t\right)^{1 / \alpha^{\prime}} & \\
& \times\left(\int_{s_{1}}^{s_{2}} \int_{U}\left|\varrho^{m} \psi\right|^{2(d+2) / d} d x d t\right)^{1 / \alpha} .
\end{aligned}
$$

Applying the inequality $x y \leq \varepsilon x^{\alpha}+C(\alpha, \varepsilon) y^{\alpha^{\prime}}$, with $\varepsilon>0$ sufficiently small, we obtain the assertion of the theorem.

Theorem 3.4. Let $\gamma>(d+2) / 2$ and suppose that we are given balls $U$ and $U^{\prime}$ in $\mathbb{R}^{d}$ such that $\overline{U^{\prime}} \subset U$. Also let $\left[s_{1}, s_{2}\right] \subset(0, T)$. Then for all $s \in\left(s_{1}, s_{2}\right)$ there exists a number $C>0$ depending only on $U, U^{\prime}, s, s_{1}, d$, and $\gamma$ such that

$$
\|\varrho\|_{L^{\infty}\left(U^{\prime} \times\left[s, s_{2}\right]\right)} \leq C\left(1+\lambda^{-1}\right)^{\gamma} \int_{s_{1}}^{s_{2}} \int_{U}\left(1+\|A\|^{\gamma}+\left|c^{+}\right|^{\gamma}+\left|\sqrt{A^{-1}} B\right|^{2 \gamma}\right) \varrho d x d t,
$$

where $\lambda=\lambda\left(U,\left[s_{1}, s_{2}\right]\right)$ and $\|A\|$ are defined above.

Proof. If $\varrho \equiv 0$ on $U \times\left[s_{1}, s_{2}\right]$, then the assertion is trivial. Consider the case where $\varrho \neq \equiv 0$. By multiplying the solution $\varrho$ by the number

$$
\left(1+\lambda^{-1}\right)^{-\gamma}\left(\int_{s_{1}}^{s_{2}} \int_{U}\left(1+\|A\|^{\gamma}+\left|c^{+}\right|^{\gamma}+\left|\sqrt{A^{-1}} B\right|^{2 \gamma}\right) \varrho d x d t\right)^{-1}
$$

we can assume that

$$
\left(1+\lambda^{-1}\right)^{\gamma} \int_{s_{1}}^{s_{2}} \int_{U}\left(1+\|A\|^{\gamma}+\left|c^{+}\right|^{\gamma}+\left|\sqrt{A^{-1}} B\right|^{2 \gamma}\right) \varrho d x d t=1 .
$$

In this case, to prove the theorem it is sufficient to find a number $C$ depending only on $U, U^{\prime}, s, s_{1}, s_{2}, d$, and $\gamma$ such that

$$
\|\varrho\|_{L^{\infty}\left(U^{\prime} \times\left[s, s_{2}\right]\right)} \leq C .
$$

Let $U=U\left(x_{0}, R\right), U^{\prime}=U\left(x_{0}, R^{\prime}\right)$, and $R^{\prime}<R$. We set $R_{n}=R^{\prime}+\left(R-R^{\prime}\right) 2^{-n}$, $s_{n}=s-\left(s-s_{1}\right) 2^{-n}$, and $U_{n}=U\left(x_{0}, R_{n}\right)$. We consider the system of nested cylinders

$$
Q_{n}=U_{n} \times\left[s_{n}, s_{2}\right], \quad Q_{0}=U \times\left[s_{1}, s_{2}\right] .
$$

For every $n$ we define a function $\psi_{n} \in C_{0}^{\infty}\left(\mathbb{R}^{d} \times(0, T)\right)$ in the same way as in the proof of Theorem 3.3. that is, $\psi(x, t)=1$ for $(x, t) \in Q_{n+1}, 0 \leq \psi \leq 1$, the support of $\psi$ is contained in $U_{n} \times\left(s_{n}, T\right)$, and $\left|\partial_{t} \psi_{n}(x, t)\right|+\left|\nabla \psi_{n}(x, t)\right| \leq K^{n}$ for all $(x, t) \in \mathbb{R}^{d}$ and some number $K>1$ depending only on the numbers $s, s_{1}, R, R^{\prime}$. 
Applying Lemma 3.2 and Hölder's inequality with exponents $\gamma$ and $\gamma^{\prime}$ we obtain

$$
\left(\int_{Q_{n}}\left|\varrho^{m} \psi_{n}\right|^{2(d+2) / d} d x d t\right)^{d /(d+2)} \leq 32 m^{2} C(d, s) K^{2 n}\left(\int_{Q_{n}} \varrho^{(2 m-1) \gamma^{\prime}+1} d x d t\right)^{1 / \gamma^{\prime}} \text {. }
$$

We set

$$
p_{n+1}=\beta p_{n}+\left(\gamma^{\prime}-1\right) \gamma^{\prime-1}, \quad p_{1}=\gamma^{\prime}+1, \quad \beta=(d+2) d^{-1} \gamma^{\prime-1} .
$$

We observe that $\beta^{n-1} p_{1} \leq p_{n} \leq \beta^{n-1}\left(p_{1}+1\right)$. Setting $m=p_{n+1} d /(2 d+4)$ we arrive at the inequality

$$
\|\varrho\|_{L^{p_{n+1}}\left(Q_{n+1}\right)} \leq C^{n \beta^{-n}}\|\varrho\|_{L^{p_{n}}\left(Q_{n}\right)}^{p_{n} /\left(p_{n}+\gamma^{\prime}-1\right)},
$$

where $C$ depends only on $K, d$ and $\gamma$. Note that $\sum_{n} n \beta^{-n}<\infty$. Furthermore, Theorem 3.3 gives an estimate for the norm $\|\varrho\|_{L^{p_{1}}\left(Q_{1}\right)}$ in terms of a constant depending only on the numbers $p_{1}, d, s, s_{1}, U$, and $U_{1}$. Consequently, we obtain an estimate for the norms $\|\varrho\|_{L^{p_{n+1}}\left(Q_{n+1}\right)}$ that is uniform in $n$, and this gives an $L^{\infty}$-estimate.

Remark 3.5. (i) Note that the constant $C$ in Theorems 3.3 and 3.4 is independent of the number $s_{2}$.

(ii) If $c \leq 0$, then all the estimates obtained above remain valid without the coefficient $c$ on the right-hand side.

Corollary 3.6. Let $\gamma>(d+2) / 2, \kappa>0$, and $t_{0} \in(0, T)$. Then there exists $C>0$ depending only on $\kappa, t_{0}, d, \gamma$ such that for all $(x, t) \in \mathbb{R}^{d} \times\left(t_{0}, T\right)$ the estimate

$$
\varrho(x, t) \leq C\left(1+\lambda^{-1}(x, t)\right)^{\gamma} \int_{t_{0} / 2}^{t} \int_{U(x, \kappa)}\left(1+\|A\|^{\gamma}+\left|c^{+}\right|^{\gamma}+\left|\sqrt{A^{-1}} B\right|^{2 \gamma}\right) \varrho d y d \tau
$$

holds, where

$$
\lambda(x, t)=\inf \left\{(A(y, \tau) \xi, \xi):|\xi|=1,(y, \tau) \in U(x, \kappa) \times\left[t_{0} / 2, t\right]\right\} .
$$

In particular, if $\mu_{t}(d x)=\varrho(x, t) d x$ is a sub-probability measure for almost all $t \in(0, T)$, the functions $\|A\|^{\gamma},\left|c^{+}\right|^{\gamma},|B|^{2 \gamma}$ belong to $L^{1}\left(\mathbb{R}^{d} \times\left(t_{0} / 2, T\right), \mu\right)$, and the function $\|A\|^{-1}$ is uniformly bounded, then $\varrho \in L^{\infty}\left(\mathbb{R}^{d} \times\left(t_{0}, T\right)\right)$.

Proof. We shift the point $x$ to 0 and apply Theorem 3.4 for the balls $U=U(x, \kappa)$, $U^{\prime}=U(x, \kappa / 2)$ and the points $s_{1}=t_{0} / 2, s=t_{0}, s_{2}=t$.

Corollary 3.7. Let $\gamma>(d+2) / 2$ and $\Theta \in(0,1)$. Then there exists a number $C>0$ depending only on $\gamma, d$ and $\Theta$ such that for all $(x, t) \in \mathbb{R}^{d} \times(0, T)$ the estimate

$$
\begin{aligned}
\varrho(x, t) \leq C\left(1+\lambda^{-1}(x, t)\right)^{\gamma} t^{-(d+2) / 2} & \\
& \times \int_{\Theta t}^{t} \int_{U(x, \sqrt{t})}\left(1+\|A\|^{\gamma}+t^{2 \gamma}\left|c^{+}\right|^{\gamma}+t^{2 \gamma}\left|\sqrt{A^{-1}} B\right|^{2 \gamma}\right) \varrho d y d \tau
\end{aligned}
$$

holds, where

$$
\lambda(x, t)=\inf \{(A(y, \tau) \xi, \xi):|\xi|=1,(y, \tau) \in U(x, \sqrt{t}) \times[\Theta t, t]\} .
$$

In particular, if $\mu_{t}(d x)=\varrho(x, t) d x$ is a sub-probability measure for almost all $t \in(0, T)$, the functions $\|A\|^{\gamma},\left|c^{+}\right|^{\gamma},|B|^{2 \gamma}$ belong to $L^{1}\left(\mathbb{R}^{d} \times(0, T), \mu\right)$, and the function $\|A\|^{-1}$ is uniformly bounded, then there exists a number $\widetilde{C}>0$ such that

$$
\varrho(x, t) \leq \widetilde{C} t^{-d / 2} \quad \text { for all }(x, t) \in \mathbb{R}^{d} \times(0, T) .
$$

Proof. To obtain the required estimate at a point $\left(x_{0}, t_{0}\right)$ it suffices to make the change of coordinates $x \mapsto\left(x-x_{0}\right) / \sqrt{t_{0}}$ and $t \mapsto t / t_{0}$, and to apply Theorem 3.4 with $U=U(0,1)$, $U^{\prime}=U(0,1 / 2)$ and $s_{1}=\Theta, s=(1+\Theta) / 2, s_{2}=1$. 
Corollary 3.8. Let $\Phi \in C^{2,1}\left(\mathbb{R}^{d} \times(0, T)\right)$ and $\Phi>0$. Let

$$
\widetilde{c}=c+\left(\partial_{t} \Phi+\operatorname{div}(A \nabla \Phi)+B \nabla \Phi\right) \Phi^{-1}, \quad \widetilde{B}=B+\Phi^{-1} A \nabla \Phi .
$$

Let $\gamma>(d+2) / 2$ and $\Theta \in(0,1)$. Then there exists a number $C>0$ depending only on $\gamma, d, \Theta$ such that, for all $(x, t) \in \mathbb{R}^{d} \times(0, T)$,

$$
\begin{aligned}
\varrho(x, t) \leq C & \Phi(x, t)^{-1}\left(1+\lambda^{-1}(x, t)\right)^{\gamma} \\
& \times t^{-(d+2) / 2} \int_{\Theta t}^{t} \int_{U(x, \sqrt{t})}\left(1+\|A\|^{\gamma}+t^{2 \gamma}\left|\widetilde{c}^{+}\right|^{\gamma}+t^{2 \gamma}\left|\sqrt{A^{-1}} \widetilde{B}\right|^{2 \gamma}\right) \Phi \varrho d y d \tau,
\end{aligned}
$$

where $\lambda$ is defined in the preceding corollaries. In particular, if

$$
\sup _{t \in(0, T)} \int_{\mathbb{R}^{d}} \Phi(x, t) \varrho(x, t) d x<\infty,
$$

the functions $\|A\|^{\gamma} \Phi,\left|\widetilde{c}^{+}\right|^{\gamma} \Phi,|\widetilde{B}|^{2 \gamma} \Phi$ belong to $L^{1}\left(\mathbb{R}^{d} \times(0, T), \mu\right)$, and the function $\|A\|^{-1}$ is uniformly bounded, then there exists a number $\widetilde{C}>0$ such that

$$
\varrho(x, t) \leq \widetilde{C} t^{-d / 2} \Phi(x, t)^{-1} \quad \text { for all }(x, t) \in \mathbb{R}^{d} \times(0, T) .
$$

Proof. It suffices to observe that the function $\Phi \varrho$ satisfies equation (3.1) with the new coefficients $\widetilde{c}$ and $\widetilde{B}$.

We consider two typical examples which apply the results we have obtained. Suppose that $c \leq 0$ and $\mu_{t}(d x)=\varrho(x, t) d x$ is a sub-probability solution of the Cauchy problem for equation (1.1) with initial condition $\nu$ such that $|c| \in L^{1}(\mu)$ and

$$
\mu_{t}\left(\mathbb{R}^{d}\right) \leq \nu\left(\mathbb{R}^{d}\right)+\int_{0}^{t} \int_{\mathbb{R}^{d}} c(x, s) \mu_{s}(d x) d s .
$$

We obtain upper estimates for the density $\varrho$ in various situations.

Example 3.9. Let $\alpha>0, r>2$, and $k>r$. Suppose that $c \leq 0$ and

$$
\begin{array}{r}
\alpha r|x|^{r-2} \operatorname{tr} A(x, t)+\alpha r(r-2)|x|^{r-4}(A(x, t) x, x)+\alpha^{2} r^{2}|x|^{2 r-4}(A(x, t) x, x) \\
+\alpha r|x|^{r-2}(b(x, t), x)+c(x, t) \leq C-C|x|^{k}
\end{array}
$$

for some $C>0$ and all $(x, t) \in \mathbb{R}^{d} \times(0, T)$. Suppose also that the inequalities

$$
\begin{aligned}
C_{1} \exp \left(-\kappa_{1}|x|^{r-\delta}\right) \leq\|A(x, t)\| & \leq C_{2} \exp \left(\kappa_{2}|x|^{r-\delta}\right), \\
\left|b^{i}(x, t)\right|+\left|\partial_{x_{j}} a^{i j}(x, t)\right| & \leq C_{3} \exp \left(\kappa_{3}|x|^{r-\delta}\right)
\end{aligned}
$$

hold for all $(x, t) \in \mathbb{R}^{d} \times(0, T)$, where $C_{1}, C_{2}, C_{3}, \kappa_{1}, \kappa_{2}, \kappa_{3}$ are positive numbers and $\delta \in(0, r)$. Let $\alpha^{\prime} \in(0, \alpha)$. Then the density $\varrho$ satisfies the estimate

$$
\varrho(x, t) \leq C_{4} \exp \left(-\alpha^{\prime}|x|^{r}\right) \exp \left(C_{5} t^{-r /(k-r)}\right)
$$

for all $(x, t) \in \mathbb{R}^{d} \times(0, T)$ and some positive numbers $C_{4}$ and $C_{5}$.

Proof. By Example 2.7 we have the inequality

$$
\int_{\mathbb{R}^{d}} \exp \left(\alpha|x|^{r}\right) \mu_{t}(d x) \leq \gamma_{1} \exp \left(\gamma_{2} t^{-r /(k-r)}\right)
$$

for almost all $t \in(0, T)$ and some numbers $\gamma_{1}$ and $\gamma_{2}$. We set $\Phi(x)=\exp \left(\alpha^{\prime}|x|^{r}\right)$. We observe that $\widetilde{c}^{+} \leq \gamma_{3}$ for some number $\gamma_{3}$ and

$$
\left(1+\|A\|^{\gamma}+t^{2 \gamma}\left|\sqrt{A^{-1}} \widetilde{B}\right|^{2 \gamma}\right) \Phi \leq \gamma_{4} \exp \left(\alpha|x|^{r}\right)
$$

for all $(x, t) \in \mathbb{R}^{d} \times(0, T)$. The required estimate is now given by Corollary 3.8 . 
Example 3.10. Let $r>2, k>2, \gamma>d+2, \alpha>0$, and $\beta>r /(k-2)$. Suppose that the inequality

$$
\begin{aligned}
& \alpha r \operatorname{tr} A(x, t)+\alpha r(r-2)|x|^{-2}(A(x, t) x, x)+\alpha r(b(x, t), x) \\
& \quad+|x|^{2} c(x, t)+\alpha^{2} r^{2}|x|^{r-2}(A(x, t) x, x) \leq C-C|x|^{k}
\end{aligned}
$$

holds for all $(x, t) \in \mathbb{R}^{d} \times(0, T)$ and some number $C>0$. Suppose also that

$$
\begin{aligned}
C_{1}\left(1+|x|^{m / \gamma}\right)^{-1} \leq\|A(x, t)\| & \leq C_{2}\left(1+|x|^{m / \gamma}\right), \\
\left|b^{i}(x, t)\right|^{2 \gamma}+\left|\partial_{x_{j}} a^{i j}(x, t)\right|^{2 \gamma} & \leq C_{3}\left(1+|x|^{m}\right)
\end{aligned}
$$

for all $(x, t) \in \mathbb{R}^{d} \times(0, T)$, where $C_{1}, C_{2}, C_{3}$ are positive numbers and $m \geq \gamma \max \{r-$ $\left.1, r \beta^{-1}\right\}$. Let $\alpha^{\prime} \in(0, \alpha)$. Then the density $\varrho$ satisfies the estimate

$$
\varrho(x, t) \leq C_{4} t^{-(8 m \beta+r d-4 \gamma r) /(2 r)} \exp \left(-\alpha^{\prime} t^{\beta}|x|^{r}\right)
$$

for all $(x, t) \in \mathbb{R}^{d} \times(0, T)$ and some positive numbers $C_{4}$ and $C_{5}$.

Proof. By Example 2.8,

$$
\int_{\mathbb{R}^{d}} \exp \left(\alpha t^{\beta}|x|^{r}\right) \mu_{t}(d x) \leq \gamma_{1}
$$

for almost all $t \in(0, T)$ and some number $\gamma_{1}$. Note that $|x|^{p} \leq \gamma_{2} t^{-\beta p / r} \exp \left(\varepsilon t^{\beta}|x|^{r}\right)$ for all $p \geq 1$ and $\varepsilon>0$. Thus, we can apply Corollary 3.8 with $\Phi(x, t)=\exp \left(\alpha t^{\beta}|x|^{r}\right)$.

\section{ACKNOWLEDGEMENTS}

The author is deeply grateful to V. I. Bogachëv for fruitful discussions and remarks.

\section{REFERENCES}

[1] V. I. Bogachëv, N. V. Krylov, and M. Rëkner, Elliptic and parabolic equations for measures, Uspekhi Mat. Nauk 64 (2009), no. 6, 5-116; English transl., Russian Math. Surveys 64 (2009), no. 6, 9731078. MR2640966(2011c:35592)

[2] V. I. Bogachëv, M. Rëkner, and S. V. Shaposhnikov, Global regularity and estimates for solutions of parabolic equations, Teor. Veroyatn. Primen. 50 (2005), no. 4, 652-674; English transl., Theory Probab. Appl. 50 (2006), no. 4, 561-581. MR2331982 (2008f:60073)

[3] V. I. Bogachëv, M. Rëkner, and S. V. Shaposhnikov, Estimates for the densities of stationary distributions and transition probabilities of diffusion processes, Teor. Veroyatn. Primen. 52 (2007), no. 2, 240-270; English transl., Theory Probab. Appl. 52 (2008), no. 2, 209-236. MR2742501 (2011i:60143)

[4] V. I. Bogachav, M. Rëkner, and S. V. Shaposhnikov, Positive densities of transition probabilities of diffusion processes, Teor. Veroyatn. Primen. 53 (2008), no. 2, 213-239; English transl., Theory Probab. Appl. 53 (2009) no. 2, 194-215.

[5] F. O. Porper and S. D. Ėŭdel'man, Two-sided estimates of the fundamental solutions of second-order parabolic equations and some applications of them, Uspekhi Mat. Nauk 39 (1984), no. 3, 107-156; English transl., Russian Math. Surveys 39 (1984), no. 3, 119-178. MR747792 (86b:35078)

[6] S. V. Shaposhnikov, Regularity and qualitative properties of solutions of parabolic equations for measures, Teor. Veroyatn. Primen. 56 (2011), no. 2, 318-350; English transl., Theory Probab. Appl. 56 (2012), no. 2, 252-279.

[7] S. V. Shaposhnikov, Estimates for solutions of parabolic equations for measures, Dokl. Akad. Nauk 434 (2010), no. 4, 454-458; English transl., Dokl. Math. 82 (2010), no. 2, 769-772. MR2778983 (2011m:35394)

[8] D. G. Aronson and J. Serrin, Local behavior of solutions of quasilinear parabolic equations, Arch. Rational Mech. Anal. 25 (1967), 81-122. MR0244638(39:5952)

[9] A. Aibeche, K. Laidoune, and A. Rhandi, Time dependent Lyapunov functions for some Kolmogorov semigroups perturbed by unbounded potentials, Arch. Math. (Basel) 94 (2010), no. 6, 565-577. MR:2653673 (2012g:47131)

[10] V. I. Bogachev, G. Da Prato, and M. Röckner, On parabolic equations for measures, Comm. Partial Differential Equations 33 (2008), no. 1-3, 397-418. MR.2398235 (2009a:35103) 
[11] V. I. Bogachev, N. V. Krylov, and M. Röckner, On regularity of transition probabilities and invariant measures of singular diffusions under minimal conditions, Comm. Partial Differential Equations 26 (2001), no. 11-12, 2037-2080. MR 1876411 (2002m:60117)

[12] V. I. Bogachev, M. Röckner, and S. V. Shaposhnikov, On uniqueness problems related to the FokkerPlanck-Kolmogorov equation for measures. Problems in mathematical analysis. No. 61. J. Math. Sci. (N.Y.) 179 (2011), no. 1, 7-47. MR3014097

[13] K. Laidoune, G. Metafune, D. Pallara, A. Rhandi, Global properties of transition kernels associated with second-order elliptic operators, Parabolic Problems, 2011 (Progress Nonlinear Differential Equations Appl. vol. 80), Birkhäuser/Springer Basel AG, Basel, 2011, 415-432. MR3052589

[14] G. Metafune, D. Pallara, and A. Rhandi, Global properties of transition probabilities of singular diffusions, Teor. Veroyatn. Primen. 54 (2009), no. 1, 116-148; English transl., Theory Probab. Appl. 54 (2010), no. 1, 68-96. MR2766650 (2011m:35144)

[15] J. Moser, A Harnack inequality for parabolic differential equations, Comm. Pure Appl. Math. 17 (1964), 101-134. MR0159139(28:2357)

[16] C. Spina, Kernel estimates for a class of Kolmogorov semigroups, Arch. Math. (Basel) 91 (2008), no. 3, 265-279. MR2439601(2009h:35182)

Moscow State University

E-mail address: starticle@mail.ru

Translated by E. KHUKHRO

Originally published in Russian 